\title{
The graded redefined assessment of strength sensibility and prehension (GRASSP): Reliability and validity
}

Kalsi-Ryan, S ; Beaton, D ; Curt, A ; Duff, S ; Popovic, M R ; Rudhe, C ; Fehlings, M G ; Verrier, M C

\begin{abstract}
Abstract With the advent of new interventions targeted at both acute and chronic spinal cord injury (SCI), it is critical that techniques and protocols are developed that reliably evaluate changes in upper limb impairment/function. The Graded Redefined Assessment of Strength Sensibility and Prehension (GRASSP) protocol, which includes five subtests, is a quantitative clinical upper limb impairment measure designed for use in acute and chronic cervical SCI. The objectives of this study were to: (1) establish the inter-rater and test-retest reliability, and (2) establish the construct and concurrent validity with the International Standards of Neurological Classification of Spinal Cord Injury (ISNCSCI), Spinal Cord Independence Measure II (SCIM), and the Capabilities of Upper Extremity Questionnaire (CUE). The study protocol included repeated administration of the GRASSP to a cross-section of individuals with tetraplegia who were neurologically stable $(n=72)$. ISNCSCI, CUE, and SCIM assessments were also administered. Two assessors examined the individuals over a 7-day period. Reliability was tested with intra-class correlation coefficients; construct validity was established with agreement/discordance analysis between the GRASSP and ISNCSCI sensory and motor items; and concurrent validity was tested with Spearman correlation coefficients. Inter-rater and test-retest reliability for all subtests within the GRASSP were above the hypothesized value of 0.80 (0.84-0.96 and 0.86-0.98, respectively). The GRASSP is about $50 \%$ more sensitive (construct validity) than the ISNCSCI when defining sensory and motor integrity of the upper limb; the subtests showed concurrence with the SCIM, SCIM self-care subscale, and CUE. The strongest concurrence to impairment was with self-perception of function (CUE) (0.57$0.83, \mathrm{p}<0.0001)$. The GRASSP was found to demonstrate reliability, construct validity, and concurrent validity for use as a standardized upper limb impairment measure for individuals with tetraplegia.
\end{abstract}

DOI: https://doi.org/10.1089/neu.2010.1504

Posted at the Zurich Open Repository and Archive, University of Zurich

ZORA URL: https://doi.org/10.5167/uzh-54781

Journal Article

Published Version

Originally published at:

Kalsi-Ryan, S; Beaton, D; Curt, A; Duff, S; Popovic, M R; Rudhe, C; Fehlings, M G; Verrier, M C (2012). The graded redefined assessment of strength sensibility and prehension (GRASSP): Reliability and validity. Journal of Neurotrauma, 29(5):905-914.

DOI: https://doi.org/10.1089/neu.2010.1504 


\title{
The Graded Redefined Assessment of Strength Sensibility and Prehension (GRASSP): Reliability and Validity
}

\author{
Sukhvinder Kalsi-Ryan,,3,7,8 Dorcas Beaton, 2,3,7,10 Armin Curt, $^{12}$ Susan Duff,' Milos R. Popovic, 3,5,6,7,9 \\ Claudia Rudhe, ${ }^{12}$ Michael G. Fehlings, ${ }^{4,6-8}$ and Mary C. Verrier ${ }^{1,3,6,7,9}$
}

\begin{abstract}
With the advent of new interventions targeted at both acute and chronic spinal cord injury (SCI), it is critical that techniques and protocols are developed that reliably evaluate changes in upper limb impairment/function. The Graded Redefined Assessment of Strength Sensibility and Prehension (GRASSP) protocol, which includes five subtests, is a quantitative clinical upper limb impairment measure designed for use in acute and chronic cervical SCI. The objectives of this study were to: (1) establish the inter-rater and test-retest reliability, and (2) establish the construct and concurrent validity with the International Standards of Neurological Classification of Spinal Cord Injury (ISNCSCI), Spinal Cord Independence Measure II (SCIM), and the Capabilities of Upper Extremity Questionnaire (CUE). The study protocol included repeated administration of the GRASSP to a cross-section of individuals with tetraplegia who were neurologically stable $(n=72)$. ISNCSCI, CUE, and SCIM assessments were also administered. Two assessors examined the individuals over a 7-day period. Reliability was tested with intra-class correlation coefficients; construct validity was established with agreement/discordance analysis between the GRASSP and ISNCSCI sensory and motor items; and concurrent validity was tested with Spearman correlation coefficients. Inter-rater and test-retest reliability for all subtests within the GRASSP were above the hypothesized value of 0.80 (0.84-0.96 and 0.86-0.98, respectively). The GRASSP is about 50\% more sensitive (construct validity) than the ISNCSCI when defining sensory and motor integrity of the upper limb; the subtests showed concurrence with the SCIM, SCIM self-care subscale, and CUE. The strongest concurrence to impairment was with self-perception of function (CUE) $(0.57-0.83, p<0.0001)$. The GRASSP was found to demonstrate reliability, construct validity, and concurrent validity for use as a standardized upper limb impairment measure for individuals with tetraplegia.
\end{abstract}

Key words: impairment; outcome measures; tetraplegia; upper limb

\section{Introduction}

$\mathbf{U}$ PPER EXTREMITY FUNCTION is a critical determinant of independence for people with cervical spinal cord injury (SCI; Anderson, 2004; DeVivo, 1997). Small gains in upper extremity function can significantly enhance the quality of life (Anderson, 2004; Snoek et al., 2005) for this population. Thus there are many efforts focused on the development of methods to restore upper limb function after SCI (Popovic et al., 2006; Prochazka et al., 1997; Taylor et al., 2002).

Scientists conducting research in SCI report that there are few adequate outcome measures to assess upper limb impairment after cervical SCI (Dunn et al., 2008; Miller et al., 2008; van Tuijl et al., 2002). The International Standards of
Neurological Classification of Spinal Cord Injury (ISNCSCI; Marino, 2000) is the current standard used to assess neurological recovery after SCI by designating the most normal caudal level of sensory and motor spinal cord innervation. However, the ISNCSCI classifies by normal spinal cord level and in doing so, may be relatively insensitive to measure upper limb impairment in a manner that can quantify subtle changes. Hence, without sensitive measures to sufficiently document outcome in tetraplegia the efficacy of novel interventions will remain questionable (Ellaway et al., 2010; Fujiwara et al., 1999; Kohlmeyer et al., 1996; Prochazka et al., 1997). Currently, there is no well accepted outcome instrument that captures the sensory, motor, and functional upper extremity changes in a manner that will be sensitive to small

\footnotetext{
${ }^{1}$ Department of Physical Therapy, ${ }^{2}$ Department of Occupational Therapy, ${ }^{3}$ Graduate Department of Rehabilitation Science, ${ }^{4}$ Department of Surgery, ${ }^{5}$ Institute of Biomaterials and Biomedical Engineering, ${ }^{6}$ Neuroscience Program, ${ }^{7}$ University of Toronto, Toronto, Ontario, Canada. ${ }^{8}$ Krembil Neuroscience Centre, University Health Network, ${ }^{9}$ Toronto Rehabilitation Institute, Toronto, Ontario, Canada.

${ }^{10}$ St. Michael's Hospital, Mobility Evaluation and Clinical Research Unit, ${ }^{11}$ Thomas Jefferson University, Philadelphia, and ${ }^{12}$ Spinal Cord Injury Centre, University Hospital Balgrist, Zurich, Zurich, Switzerland.
} 
neurological changes over the natural course of recovery, or as a result of therapeutic intervention. The need for a sensitive outcome measure to assess upper extremity impairment, function, and recovery in patients with cervical SCI is increasingly important for two reasons. First, because nearly $50-60 \%$ of cases of SCI are incomplete and demonstrate greater potential for neurological recovery (Marino et al., 1999; Sekhon and Fehlings, 2001). Second, novel interventions aimed at enhancing neurological recovery require outcomes that can substantiate these gains in order to prove efficacy (Steeves et al., 2007).

Given this background, we developed the Graded Redefined Assessment of Strength Sensibility and Prehension (GRASSP), which is a clinical impairment measure that incorporates three domains vital to upper limb function: sensation, strength, and prehension. Theoretical and clinimetric development of the GRASSP, including the rationale and analysis used to determine inclusion of subtests, has been reported previously (Kalsi-Ryan et al., 2009). The present study focused on the following objectives: (1) to establish the inter-rater and test-retest reliability of the subtests within the GRASSP; (2) to establish the construct validity (agreement and discordance of GRASSP) against the ISNCSCI, including the American Spinal Injury Association's Classification of Spinal Cord Injury (AIS); and (3) to establish the concurrent validity of the GRASSP with the Spinal Cord Independence Measure II (SCIM) (Catz and Itzkovich, 2007; Catz et al., 2004), and Capabilities of Upper Extremity Questionnaire (CUE) (Marino et al., 1998). In this study construct validity is defined by specifying the factors that account for the variance in responses of the GRASSP in comparison to a current standard in the field (ISNCSCI). Concurrent validity is defined by the correspondence between the GRASSP and other similar measures in the field. The objective of this study was to establish psychometric properties of a new measure (GRASSP version 1.0).

\section{Background of the GRASSP version 1.0}

A theoretical framework was developed to guide the process of tool development. Item generation was based on concepts related to anatomy, physiology, SCI pathology, and function (Kalsi-Ryan et al., 2009; Brand and Hollister, 1991; Shumway-Cook and Woollacott, 2007). In order to produce a clinical measure with greater precision than existing tools, specific considerations were given when generating the items of the GRASSP. It was established by consensus that a single test would not be sufficient to meet the criteria set out for GRASSP by the Research and Design Team. Rather a battery of tests would be more appropriate for the thorough assessment of the upper limbs. Key test locations that represented significant anatomical levels of sensory innervation and functionally important areas of the hand were selected and tested with Semmes-Weinstein monofilaments (SWM). The use of SWM is a well-established sensory testing approach that demonstrates excellent psychometric properties (Mackin et al., 2003). Strength testing included muscles that had a strong representation at each anatomical neurological level (Moore, 1985), and multiple muscles per myotome were included. Traditional motor grading (Daniels and Worthington, 1995; Brandsma et al., 1995) was employed for the strength testing. Prehension was included to represent the influence of sensation and strength on goal-oriented upper limb tasks. To ensure that the presence or absence of movement of the hand during the early stages post-injury was not missed, a prehension ability test involving three types of grasps was incorporated. A prehension performance test was incorporated to make certain that movement was assessed within a functional paradigm, and how the movements were performed was evaluated. Table 1 summarizes the contents (domains/subtests), the origin of tests included, brief general instructions for administration, and the scoring scheme for the GRASSP version 1.0.

\section{Methods}

A cross-sectional multi-center trial was conducted to establish the reliability and validity of the GRASSP. The methodology of the study was focused on validating the measure among raters and against existing tests. Ethical approval was attained at all institutions participating in the study.

\section{Inclusion and exclusion criteria}

Individuals with chronic (more than 6 months after injury) traumatic tetraplegia who were neurologically and medically stable, between the ages of 16 and 65 and able to provide informed consent were included in the study. Individuals with moderate brain injury who were neurologically unstable or individuals with any other pathology causing upper limb impairment were excluded. Sample size was determined with power contours established by Doner and Eliasiw (1987). The contour used to estimate the sample size was based on a Type 1 error rate (alpha) of 0.05 , a Type II error rate (beta) of 0.2 , a null hypothesis for intra-class correlation coefficient (ICC) $=$ 0.8 and tested against the alternative hypothesis for ICC greater than 0.8 . The minimum estimated sample size was 39 for 3 repeated measures.

\section{Study design}

Seven centers participated in the trial: 3 European (University Hospital Balgrist, Trauma Centre Murnau, and Hohe Worte, Bayreuth), and 4 North American (Toronto Rehabilitation Institute, Rehabilitation Institute of Chicago, G.F. Strong and Magee Rehabilitation Hospital, and Thomas Jefferson University Hospital). Each site engaged two examiners who were either occupational or physical therapists who had expertise with SCI. In total 14 examiners were involved in the study, 12 of whom were occupational therapists and two of whom were physical therapists. Two workshops (one in Europe and one in North America) were conducted to train the examiners on the study protocol and appropriate use of all study measures. Instructions and demonstration on the administration of the primary measure, GRASSP, and secondary measures, SCIM, ISNCSCI, and CUE questionnaire, were provided to all examiners. Training was provided to reduce observer variability (Wright and Feinstein, 1992). A consensus discussion was conducted to ensure consistency of protocol and test administration; however, a formal exam was not conducted.

Each site had an assigned examiner 1 and examiner 2 . The protocol conducted in Europe consisted of two examiners assessing each study participant once; during the first visit examiner 1 administered the GRASSP, and during the second visit examiner 2 administered the GRASSP a second time. Examiner 1 conducted all secondary measures on the second 


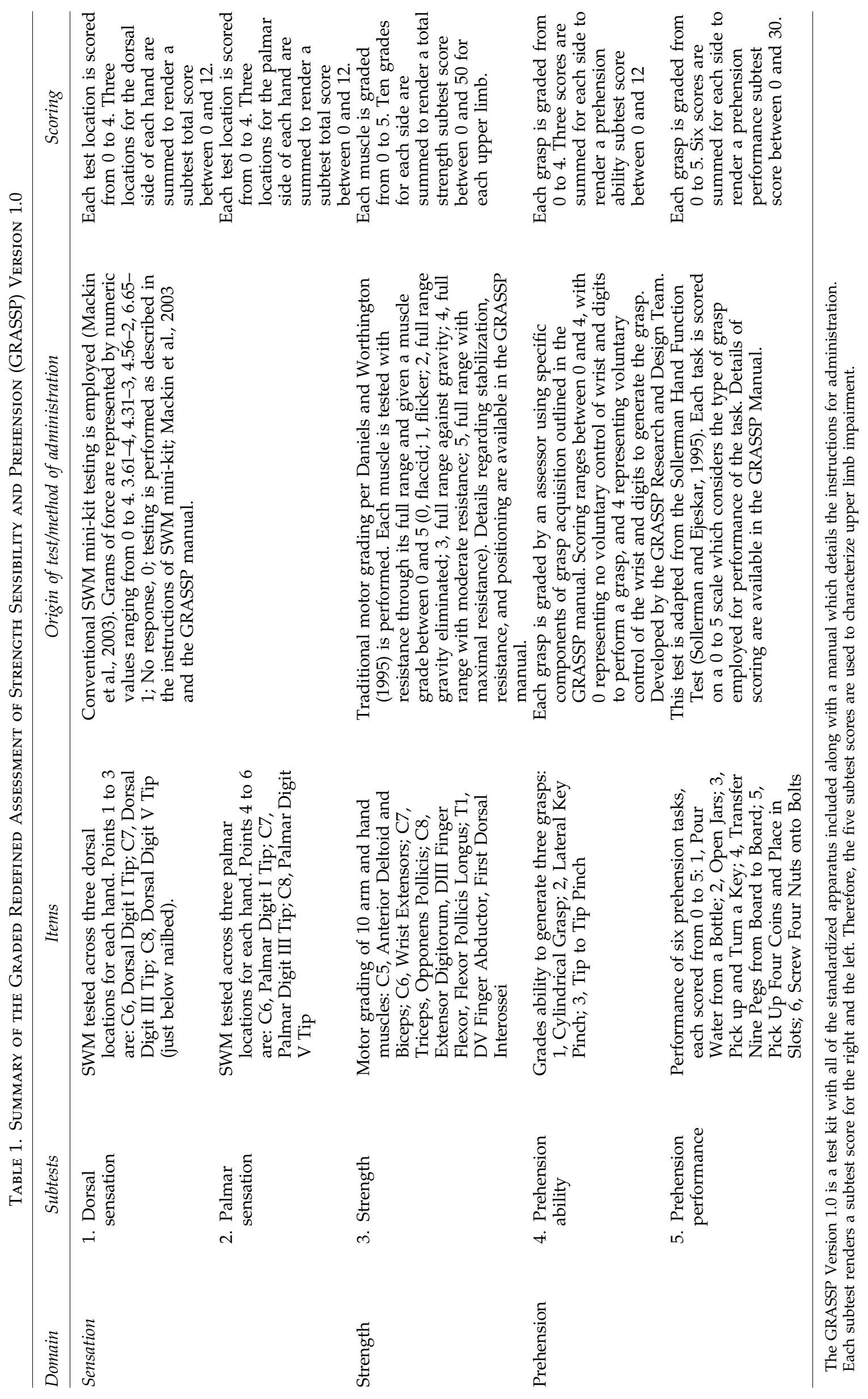




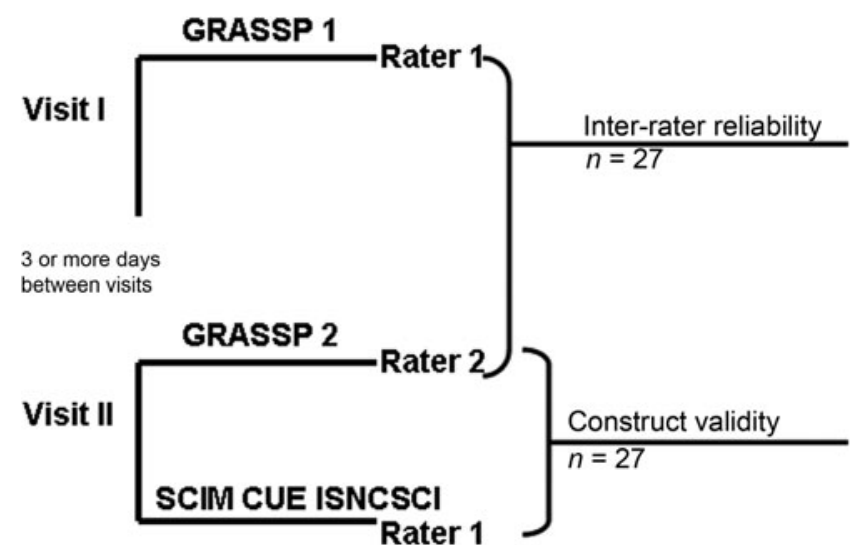

FIG. 1. Study design used to collect European data. This figure refers to the method of data collection in the European centers $(n=3)$ and how the data were used in the analysis. The general methodology for this study was based in establishing psychometric properties. Therefore, a stable sample was targeted and repeated administration of the GRASSP was conducted along with the administration of the comparator measures (SCIM, CUE, and ISNCSCI). The figure defines the visits, number of GRASSP administrations, raters, and the use of data for analysis. When compared to Figure 2 this figure defines how the study design was similar and different in North America and Europe (GRASSP, Graded Redefined Assessment of Strength Sensibility and Prehension; ISNCSCI, International Standards of Neurological Classification of Spinal Cord Injury; SCIM, Spinal Cord Independence Measure II; CUE, Capabilities of Upper Extremity Questionnaire).

visit. In Europe visits one and two were separated by at least 3 days. All test material was translated from English to German by the same individual for use in the Swiss and German centers. European data were used in the analysis to establish inter-rater reliability and validity (Fig. 1).

The protocol conducted in North America consisted of two examiners assessing each study participant a total of three times. During the first visit examiners 1 and 2 administered the GRASSP approximately $1 \mathrm{~h}$ apart, and during the second visit examiner 1 administered the GRASSP and examiner 2 administered all of the secondary measures. Visits were separated by 3-7 days. North American data were used in the analysis of inter-rater and test-retest reliability and validity (Fig. 2). The European and North American data were pooled to render 72 data sets for the analysis of inter-rater reliability and construct and concurrent validity. The 45 data sets collected at North American sites were used for the analysis of test-retest reliability. Figures 1 and 2 define which data from Europe and North America were used for inter-rater reliability analysis, which data were used for test-retest reliability, and which data were used in validity analysis. All data were de-identified, entered into Excel tables, and sent to the central site. Data were aggregated and analyzed using SAS 9.1.

\section{Outcome measures}

The outcome measures used in the study for comparison with the GRASSP version 1.0 were selected based on their use in the field of SCI assessment and/or their established quali-

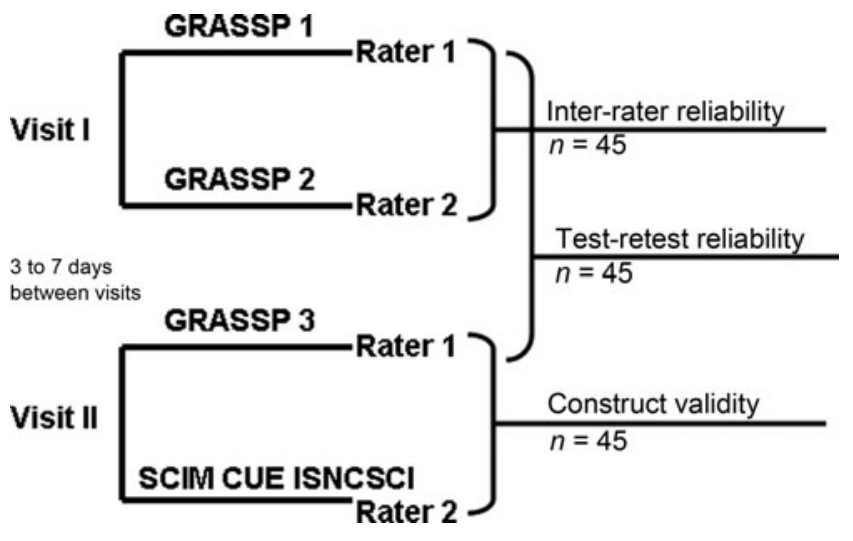

FIG. 2. Study design used to collect North American data. This figure refers to the method of data collection in the North American centers $(n=4)$ and how the data were used in the analysis. The general methodology for this study was based in establishing psychometric properties. Therefore, a stable sample was targeted and repeated administration of the GRASSP was conducted along with the administration of the comparator measures (SCIM, CUE, and ISNCSCI). The figure defines the visits, number of GRASSP administrations, raters, and the use of data for analysis. When compared to Figure 1 this figure defines how the study design was similar and different in North America and Europe (GRASSP, Graded Redefined Assessment of Strength Sensibility and Prehension; ISNCSCI, International Standards of Neurological Classification of Spinal Cord Injury; SCIM, Spinal Cord Independence Measure II; CUE, Capabilities of Upper Extremity Questionnaire).

ties. The ISNCSCI provides a sensory and motor level for each side based on the most normal caudal spinal cord level that is represented by the dermatomes and myotomes tested. A sensory or motor neurological level is derived when the most caudal side is used to express the spinal level. An SCI can also be classified according to the American Spinal Cord Injury Association (ASIA) impairment scale as A, B, C, D, or E. Four different syndromes for SCI can be defined, and finally a zone of partial preservation is derived from the partial sensory and/or motor integrity below the assigned ISNCSCI levels (Marino, 2000). Reliability is 35-93\% consistent among raters across sensory and motor testing (Priebe and Waring, 1991). The ISNCSCI was selected for use in the study to define the sample according to an international classification method and to define the severity of injury for individuals involved in the study. As the ISNCSCI is the most widely used measure to define sensory and motor levels in SCI, it is considered by some to be a "gold standard," and therefore is used as a comparator to establish validity with GRASSP version 1.0.

The SCIM is a global measure of function specific for individuals with SCI (Catz et al., 2004), and was used to define the function and independence of the sample in this study. The SCIM assesses function in three core areas: (1) self-care, which includes feeding, bathing, dressing, and grooming, and is scored between a range of 0 to 20; (2) respiration and sphincter management, which are scored between a range of 0 to 40; and (3) mobility, also scored between a range of 0 to 40 (Catz and Itzkovich, 2007). Inter-rater reliability is above 0.8 when assessed by agreement statistics for most SCIM items, and ICC for the total score is 0.94 . Concurrent validity of the 
SCIM with the Functional Independence Measure used for other populations as well as SCI is 0.79 (Catz et al., 2004). The SCIM total and self-care subscale (SCIM-SS) were used as comparator scores. The SCIM-SS includes items solely related to the use of the upper limb; therefore comparisons between the GRASSP subtests were made with the SCIM-SS rather than the total SCIM score in this analysis. The subscales of the SCIM are reliable and useful quantitative representations of the specific constructs of independence in SCI (Catz and Itzkovich, 2007). Some researchers have found the subscales to be more specific for targeted analyses, such as the mobility subscale for comparison with other measures of walking, and the self-care subscale for comparison with other measures of the upper limb (Rudhe and van Hedel, 2009; van Hedel and EMSCI Study Group, 2009).

The CUE questionnaire is a 32-item questionnaire developed to assess difficulty in performing certain activities with the upper extremities. The CUE questionnaire is a selfperceived measure of upper limb function which incorporates components of reach, grasp, and manipulation. The scoring is based on one's perception of the degree of difficultly in performing tasks, which is important in defining functional limitation (Marino et al., 1998). Psychometric properties of the CUE questionnaire have been reported as 0.92 for test-retest reliability, as tested by Cronbach's alpha, and 0.74 for concurrent validity with the Functional Independence Measure, as tested by Pearson correlation coefficient (Marino et al., 1998). The CUE questionnaire was selected as a comparator measure to establish validity and determine relationships between impairment and self-perceived function.

The ISNCSCI, although it is not specific to the upper limb, was selected because of its use as a primary outcome measure in many SCI studies, and the items specific to the upper limb were predominantly used for validation. The SCIM was selected as a measure of function and independence. The CUE questionnaire was selected as a self-perceived measure of function because consumer input in terms of an individual's own evaluation of their functional status is becoming increasingly important in the field (Anderson, 2004). Although the measures selected for validity comparison did not share all the same constructs as the GRASSP, they were the most appropriate measures available, with well-established psychometric properties.

\section{Analysis}

A priori we anticipated the following: (1) inter-rater and test-retest reliability for subtest scores would be greater than or equal to an ICC value of $r=0.80$. According to Streiner and Norman, reliability is considered to be good if the ICC is above 0.75 (Portney and Watkin, 2000; Streiner and Norman, 1995a); (2) construct validity (Patrick and Erickson, 1993; Streiner and Norman, 1995b) would be demonstrated by GRASSP sensation and strength subtests defining sensory and motor impairment with greater precision than the ISNCSCI; and (3) concurrent validity of the GRASSP would be demonstrated by a moderate and positive correlation with the SCIM, SCIM-SS, and the CUE questionnaire.

Reliability was analyzed by conducting non-parametric ICC on the GRASSP subtest total scores (Portney and Watkin, 2000). The GRASSP was designed to have a broader range of items and response levels related to the upper limb in comparison to the ISNCSCI, therefore it would be expected that the
GRASSP sensation and strength subtests and items would define the sample with greater precision. Furthermore, the ISNCSCI derives neurological levels based on the complete intactness of normal innervation and in doing so, sensory and motor integrity below the normal spinal cord level is not incorporated into the ISNCSCI sensory and motor level. Essentially, the impairment is not fully defined by the designation of a level in the ISNCSCI. GRASSP on the other hand does not derive a level from the testing. Therefore, to compare the tests, items were compared rather than levels and total scores. Construct validity was analyzed by comparing the descriptive frequency data of the ISNCSCI sensory and motor test items with the corresponding GRASSP subtest items. Instead of comparing total scores or levels derived, item comparisons were conducted to ensure that summed data did not influence the results. The individual sensory (SWM) test locations in the GRASSP were compared to the sensory test locations for light touch in the ISNCSCI. ISNCSCI sensory levels were used only to group the sample for presentation of the data; the items are presented both in subgroups and as the whole sample. All comparisons were made for the right and left sides separately. Each study participant's sensory information was compared (dorsal and palmar components from the GRASSP were combined) using three conditions of comparison: (1) agreement between GRASSP sensation and ISNCSCI sensory items (absent, impaired, or normal); (2) discordance between GRASSP sensation and ISNCSCI sensory items due to the added palmar test locations; and (3) discordance between GRASSP sensation and ISNCSCI sensory items due to the increased response levels used in the GRASSP.

Kappa coefficients were also conducted on the sensory comparisons to determine the degree of agreement and discordance between both measures. Agreement was established using the standards as established by Landis and Koch: .00, poor; .01-.20, slight; .21-.40, fair; .41-.60, moderate; .61-.80, substantial; and .81-1.00, almost perfect (Landis and Koch, 1977).

The individual muscles in the GRASSP were compared to the muscles tested in the ISNCSCI. ISNCSCI motor levels were used only to group the sample for presentation of the data; the items are presented both in subgroups and as the whole sample. All comparisons were made for right and left sides separately. Each study participant's strength information was compared and three conditions of comparison existed: (1) agreement between GRASSP strength items and ISNCSCI upper limb motor items; (2) discordance between GRASSP strength items and ISNCSCI upper limb motor items due to the added muscles in the GRASSP; and (3) discordance between GRASSP strength items and ISNCSCI upper limb motor items due to the derivation of a level from the motor testing in the ISNCSCI.

Concurrent validity was analyzed by conducting Spearman correlation coefficients between GRASSP subtest scores with SCIM total scores, SCIM-SS, and CUE questionnaire scores.

\section{Results}

\section{Sample}

The data used in this analysis included a multicentre/ multinational cross-section of data; the total sample consisted of 72 individuals with chronic tetraplegia ranging from 6 months to 20 years post-injury. Demographic data and descriptive statistics are summarized in Table 2. Distribution of the sample according to the ISNCSCI is defined in Table 3, 
Table 2. Demographic, ISNCSCi Values, Comparator Measures, and GRASSP Results for the Total Sample ( $N=72)$

\begin{tabular}{|c|c|c|c|c|c|c|c|c|}
\hline \multirow{3}{*}{$\begin{array}{l}\text { Demographics } \\
\text { Age (years) } \\
\text { Time post-injury (years) }\end{array}$} & \multicolumn{2}{|c|}{ Mean } & \multicolumn{2}{|c|}{$S D$} & \multicolumn{2}{|c|}{ Range } & \multicolumn{2}{|c|}{ Median } \\
\hline & \multicolumn{2}{|c|}{39.7} & \multicolumn{2}{|c|}{10.7} & \multicolumn{2}{|c|}{$16-65$} & \multicolumn{2}{|c|}{42} \\
\hline & \multicolumn{2}{|c|}{7.6} & \multicolumn{2}{|c|}{6.1} & \multicolumn{2}{|c|}{$0.5-20$} & \multicolumn{2}{|c|}{6.2} \\
\hline \multicolumn{9}{|l|}{ ISNCSCI } \\
\hline & $R$ & $L$ & $R$ & $L$ & $R$ & $L$ & $R$ & $L$ \\
\hline UEMS 0-25 & 14.1 & 14.3 & 5.9 & 6.3 & $2-25$ & $1-25$ & 13 & 14 \\
\hline LEMS 0-25 & 1.9 & 2.0 & 5.7 & 5.6 & $0-25$ & $0-24$ & 0 & 0 \\
\hline LT sensory score $(0-112)$ & \multicolumn{2}{|c|}{39.4} & \multicolumn{2}{|c|}{23.9} & \multicolumn{2}{|c|}{$13-103$} & \multicolumn{2}{|c|}{29} \\
\hline PP sensory score (0-112) & \multicolumn{2}{|c|}{33.1} & \multicolumn{2}{|c|}{19.6} & \multicolumn{2}{|c|}{ 9-100 } & \multicolumn{2}{|c|}{27} \\
\hline \multicolumn{9}{|l|}{ Comparator measure } \\
\hline SCIM (0-100) & \multicolumn{2}{|c|}{45.1} & \multicolumn{2}{|c|}{21.1} & \multicolumn{2}{|c|}{$11-99$} & \multicolumn{2}{|c|}{38} \\
\hline SCIM-SS (0-20) & \multicolumn{2}{|c|}{9.8} & \multicolumn{2}{|c|}{5.7} & \multicolumn{2}{|c|}{$0-20$} & \multirow{2}{*}{\multicolumn{2}{|c|}{$\begin{array}{c}9 \\
78\end{array}$}} \\
\hline CUE (0-124) & & & & & & & & \\
\hline \multicolumn{9}{|l|}{ GRASSP } \\
\hline & $R$ & $L$ & $R$ & $L$ & $R$ & $L$ & $R$ & $L$ \\
\hline Strength $(0-50)$ & 24.3 & 25.1 & 13.0 & 13.5 & $5-50$ & $1-50$ & 18 & 19 \\
\hline Dorsal sensation (0-12) & 6.5 & 6.7 & 3.2 & 3.1 & $0-12$ & $0-12$ & 6 & 6 \\
\hline Palmar sensation (0-12) & 7.1 & 7.2 & 3.6 & 3.3 & $0-12$ & $0-12$ & 7 & 7 \\
\hline Prehension ability $(0-12)$ & 4.9 & 5.1 & 4.5 & 4.3 & $0-12$ & $0-12$ & 2 & 2 \\
\hline Prehension performance $(0-30)$ & 15.6 & 14.7 & 9.6 & 8.9 & $0-30$ & $0-30$ & 12 & 12 \\
\hline
\end{tabular}

${ }^{a}$ Values based on $n=45$.

SD, standard deviation; R, right; L, left; SCIM, Spinal Cord Independence Measure II; SCIM-SS, Spinal Cord Independence Measure SelfCare Subscale: CUE, Capabilities of Upper Extremity Questionnaire; ISNCSCI, International Standards for Neurological Classification of Spinal Cord Injury; UEMS, Upper Extremity Motor Score; LEMS, Lower Extremity Motor Score; LT, Light Touch; PP, pinprick; GRASSP, Graded Redefined Assessment of Strength Sensibility and Prehension.

showing subgroups according to ISNCSCI motor and sensory levels (right and left data are presented side by side). Approximately $52.5 \%$ of the individuals fell into the C6-C7 motor levels, while approximately $66 \%$ fell into the C4-C6 sensory levels. The ISNCSCI Impairment Scale (AIS), which defines completeness of the sample, identified 39\% $(n=28)$ of the sample as complete, and $61 \%$ of the sample as incomplete.

\section{Reliability}

Inter-rater and test-retest reliability were established for each subtest total, and for the right and left sides separately. Table 4 lists the non-parametric ICC values for the subtests within the GRASSP, including the confidence interval for each

Table 3. Demographics of the Sample Based on ISNCSCI and AIS Classification $(N=72$, Presented as N Values of the Sample)

\begin{tabular}{lcrrrr}
\hline & \multicolumn{2}{c}{ Motor level } & & \multicolumn{2}{c}{ Sensory level } \\
\cline { 2 - 3 } \cline { 5 - 6 } & \multicolumn{2}{c}{$\mathrm{n}$} & & \multicolumn{2}{c}{$\mathrm{n}$} \\
\cline { 2 - 3 } \cline { 5 - 6 } ISNCSCI levels & Right & Left & & Right & Left \\
\hline C2-C4 & 10 & 14 & & 29 & 29 \\
C5 & 10 & 9 & & 11 & 9 \\
C6 & 23 & 21 & & 17 & 19 \\
C7 & 17 & 16 & & 8 & 6 \\
C8 & 4 & 5 & & 1 & 2 \\
T1 and below & 8 & 7 & & 6 & 7 \\
AIS classification & $A$ & $B$ & & $C$ & D \\
& 28 & 18 & & 12 & 14 \\
\end{tabular}

ISNCSCI, International Standards for Neurological Classification of Spinal Cord Injury; AIS, American Spinal Cord Injury Association Impairment Scale. value. ICC for inter-rater reliability ranged between 0.84 and 0.96 , and test-retest reliability ranged between 0.86 and 0.98 , with a significance level of $p<0.0001$ for all values.

\section{Construct validity}

Precision of the GRASSP was established by comparing the sensation and strength subtest items to the sensory and motor upper limb items in the ISNCSCI. It should be noted that individuals with a sensory level of $\mathrm{C} 5$ or any level rostral to $\mathrm{C} 5$ would be expected to score 0 on the sensory testing in the GRASSP. Table 5 defines the proportions of the subgroups and the whole sample that fell into three different conditions. On average $54 \%$ of the sample showed discordance in sensory innervation when assessed with the GRASSP due to the

Table 4. Reliability Values of Subtest Scores WITHIN THE GRASSP

\begin{tabular}{|c|c|c|c|c|}
\hline \multirow[b]{2}{*}{ Subtest } & \multicolumn{2}{|c|}{$\begin{array}{l}\text { Inter-rater } \\
\text { reliability }\end{array}$} & \multicolumn{2}{|c|}{$\begin{array}{l}\text { Test-retest } \\
\text { reliability }\end{array}$} \\
\hline & ICC & $C I$ & ICC & $C I$ \\
\hline Sensation right & 0.84 & $0.75-0.89$ & 0.95 & $0.91-0.97$ \\
\hline Sensation left & 0.91 & $0.86-0.94$ & 0.86 & $0.76-0.92$ \\
\hline Strength right & 0.95 & $0.93-0.97$ & 0.98 & $0.98-0.99$ \\
\hline Strength left & 0.95 & $0.92-0.97$ & 0.98 & $0.96-0.98$ \\
\hline Prehension ability right & 0.95 & $0.92-0.97$ & 0.98 & $0.96-0.99$ \\
\hline Prehension ability left & 0.95 & $0.92-0.97$ & 0.98 & $0.97-0.99$ \\
\hline Prehension performance right & 0.95 & $0.92-0.97$ & 0.93 & $0.88-0.96$ \\
\hline Prehension performance left & 0.96 & $0.93-0.97$ & 0.96 & $0.93-0.98$ \\
\hline
\end{tabular}

All values had a significance level of $p<0.0001$.

ICC, intra-class correlation coefficient; CI, confidence interval; GRASSP, Graded Redefined Assessment of Strength Sensibility and Prehension. 
Table 5. Construct Validity Agreement/Discordance of Sensory Results between GRASSP AND ISNCSCI $(N=72)$

\begin{tabular}{lrrrrr}
\hline & \multicolumn{2}{c}{ Agreement } & & \multicolumn{2}{c}{ Discordance } \\
\cline { 2 - 3 } \cline { 5 - 6 } ISNCSCI sensory level $^{\mathrm{a}}$ & $\mathrm{n}$ & $\mathrm{n}(\%)$ & & $\begin{array}{c}1 \\
\mathrm{n}(\%)\end{array}$ & $\begin{array}{c}2 \\
\mathrm{n}(\%)\end{array}$ \\
\hline Right total sample & 72 & $32(44)$ & & $16(22)$ & $24(33)$ \\
Left total sample & 72 & $34(47)$ & & $13(18)$ & $25(35)$ \\
Right C2-C4 & 29 & $14(19)$ & & $7(10)$ & $8(11)$ \\
Left C2-C4 & 29 & $12(17)$ & & $6(8)$ & $11(15)$ \\
Right C5 & 11 & $5(7)$ & & $4(6)$ & $2(3)$ \\
Left C5 & 9 & $5(7)$ & & $3(4)$ & $1(1)$ \\
Right C6 & 17 & $6(8)$ & & $5(7)$ & $6(8)$ \\
Left C6 & 19 & $8(11)$ & & $4(6)$ & $7(10)$ \\
Right C7 & 8 & $4(6)$ & & $0(0)$ & $4(6)$ \\
Left C7 & 6 & $3(4)$ & & $0(0)$ & $3(4)$ \\
Right C8 and below & 7 & $3(4)$ & & $0(0)$ & $4(6)$ \\
Left C8 and below & 9 & $6(8)$ & & $0(0)$ & $3(4)$ \\
\hline
\end{tabular}

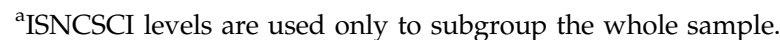

1, discordance due to added palmar test locations in GRASSP; 2, discordance due to the increased response levels (SWM) used in the GRASSP; Graded Redefined Assessment of Strength Sensibility and Prehension; ISNCSCI, International Standards of Neurological Classification of Spinal Cord Injury; SWM, Semmes-Weinstein monofilaments.

additional test locations of sensory testing included. Table 6 shows the level of agreement between ISNCSCI-light touch (ISNCSCI-LT) and GRASSP-SWM for the C6, C7, and C8 dorsal test locations. The kappa coefficients of $\mathrm{C} 6, \mathrm{C} 7$, and $\mathrm{C} 8$ reveal that the level of agreement is not substantial; the statistical analyses indicate that the two tests demonstrate different results. Essentially the ISNCSCI-LT and SWM provide different results regarding the sensory status of individuals with tetraplegia. Table 7 defines the proportions of the subgroups and the whole sample that fall into three different conditions. On average, $53 \%$ of the sample showed a different degree of motor innervation when assessed with the GRASSP due to the added muscles in the GRASSP, and the designation of most caudal level in the ISNCSCI.

Table 6. LeVel of AgReEment betweEn GRASSP-SWM AND ISNCSCI-LT FOR C6, C7, AND C8

\begin{tabular}{lccc}
\hline & \multicolumn{3}{c}{ ISNCSCI-LT } \\
\cline { 2 - 4 } GRASSP-SWM & C6 & C7 & C8 \\
\hline Right C6 & 0.412 & & \\
Left C6 & 0.442 & & \\
Right C7 & & 0.474 & \\
Left C7 & & 0.459 & 0.511 \\
Right C8 & & 0.503 \\
Left C8 & & & \\
\hline
\end{tabular}

This table presents the kappa coefficients for the comparisons made between $\mathrm{C} 6, \mathrm{C} 7$, and $\mathrm{C} 8$ test locations on the dorsal surface of the hand. The comparisons are made between the LT and SWM measurements made over the same test locations. All values have a significance level of $p<0.001$.

SWM, Semmes-Weinstein monofilaments; L, light touch; GRASSP, Graded Redefined Assessment of Strength Sensibility and Prehension; ISNCSCI, International Standards of Neurological Classification of Spinal Cord Injury.
Table 7. Construct Validity Agreement/Discordance of Strength Results between the GRASSP AND ISNCSCI $(N=72)$

\begin{tabular}{|c|c|c|c|c|}
\hline \multirow[b]{3}{*}{ ISNCSCI motor level ${ }^{\mathrm{a}}$} & \multirow{2}{*}{\multicolumn{2}{|c|}{ Agreement }} & \multicolumn{2}{|c|}{ Discordance } \\
\hline & & & \multirow{2}{*}{$\begin{array}{c}1 \\
\mathrm{n}(\%)\end{array}$} & \multirow{2}{*}{$\begin{array}{c}2 \\
\mathrm{n}(\%)\end{array}$} \\
\hline & $\mathrm{n}$ & n $(\%)$ & & \\
\hline Right total sample & 72 & $36(50)$ & $19(26)$ & $17(24)$ \\
\hline Left total sample & 72 & $34(47)$ & $20(28)$ & $17(24)$ \\
\hline Right C2-C4 & 10 & $1(1)$ & $6(8)$ & $3(4)$ \\
\hline Left C2-C4 & 14 & $6(8)$ & $6(8)$ & $1(1)$ \\
\hline Right C5 & 10 & $3(4)$ & $2(3)$ & $5(7)$ \\
\hline Left C5 & 9 & $3(4)$ & $1(1)$ & $5(7)$ \\
\hline Right C6 & 23 & $13(18)$ & $3(4)$ & $7(10)$ \\
\hline Left C6 & 21 & $11(15)$ & $5(7)$ & $4(6)$ \\
\hline Right C7 & 17 & 9 (12) & $6(8)$ & $2(3)$ \\
\hline Left C7 & 16 & $6(8)$ & $7(10)$ & $3(4)$ \\
\hline Right C8 & 4 & $2(3)$ & $2(3)$ & $0(0)$ \\
\hline Left C8 & 5 & $1(1)$ & $1(1)$ & $3(4)$ \\
\hline Right T1 and below & 8 & $8(11)$ & $0(0)$ & $0(0)$ \\
\hline Left T1 and below & 7 & $7(10)$ & $0(0)$ & $0(0)$ \\
\hline
\end{tabular}

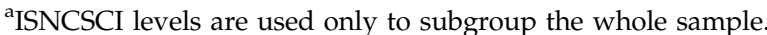

1 , discordance due to added palmar test locations in the GRASSP; 2 , discordance due to the increased response levels (SWM) used in the GRASSP; GRASSP, Graded Redefined Assessment of Strength Sensibility and Prehension; ISNCSCI, International Standards of Neurological Classification of Spinal Cord Injury; SWM, SemmesWeinstein monofilaments.

\section{Concurrent validity}

Table 8 displays all of the concurrent validity values. Right and left data were combined for the analyses, and Spearman correlation coefficients were used to establish the association between GRASSP subtests and the CUE questionnaire, SCIM total, and SCIM-SS. All associations were positive, were fair to moderate in strength, and were significant with $p$ values of less than 0.0001. The SCIM-SS showed a stronger association than the SCIM total with the subtests of the GRASSP. The CUE questionnaire showed the strongest associations with the GRASSP subtests, which signifies a strong association between self-perceived function and tested impairment.

\section{Discussion}

In order for the GRASSP to be an accepted measure for use in SCI research, psychometric testing with the tetraplegic

Table 8. Concurrent Validity GRASSP Subtests and Functional Measures

\begin{tabular}{lccc}
\hline Subtest score & SCIM & SCIM-SS & CUE \\
\hline Sensation total (R+L) & 0.57 & 0.74 & 0.77 \\
Strength total (R+L) & 0.59 & 0.74 & 0.76 \\
Prehension performance total (R+L) & 0.68 & 0.79 & 0.83 \\
\hline
\end{tabular}

All values had a significance level of $p<0.0001$; Pearson correlation coefficient: moderate concurrence $=0.61-0.79$; substantial concurrence $=0.80-1.00$.

SCIM, Spinal Cord Independence Measure II; SCIM-SS, Spinal Cord Independence Measure Self-Care Subscale; CUE, Capabilities of Upper Extremity Questionnaire; SWM, Semmes-Weinstein monofilaments; R, right; L, left; GRASSP, Graded Redefined Assessment of Strength Sensibility and Prehension. 
population was a requirement. As a result of this study, the psychometric properties of reliability and validity have been established. The GRASSP was designed to be a sensitive clinical impairment measure specific to the upper limb with a sound theoretical framework and relevant domains. The results of this study provide the evidence for a clinician or researcher to consider the use of the GRASSP in a clinical trial or clinical setting as an adjunct to existing outcome measures, particularly when hand function is of importance.

Reliability is considered good for group-level analyses when the ICC is greater than 0.75 (Streiner and Norman, 1995a), and good for individual decision making at a level of 0.90 (Nunnally and Bernstein, 1996). This study provides strong evidence to support the reliability of the GRASSP when administered by trained clinicians (ICCs ranged between .84 and .98), and repeated assessments done by the same or different examiners rendered reliable results. A change in GRASSP scores can confidently be attributed to a clinical change in impairment of the upper limb.

Two types of validity were established in this study. First, construct validity was demonstrated by using the theoretical basis to develop a measure that was able to define a broader range of findings (more sensitive), and in this particular case was broader than the current gold standard, the ISNCSCI. Greater accuracy was one of the underlying a priori requirements set for the GRASSP during the developmental phase, as it was intentionally created to provide a more accurate representation of impairment in the case of sensation. Enhanced accuracy was to be accomplished by using a more reliable sensory modality (SWM), with a greater range of response levels, and by including palmar test locations. Individuals that fall into the C2-C4 ISNCSCI sensory level group should have no normal sensation on the dorsum of the hand, which would ensure agreement of tests. However, by using a more sensitive test modality (SWM in the GRASSP), and adding palmar test locations, more sensation is noted by the GRASSP sensory testing than that reported by the ISNCSCI, particularly in the C2-C4 subgroup. A similar finding was noted with the C2-C4 ISNCSCI motor level group. This group should not have arm and hand strength, and the GRASSP strength testing noted more strength than that reported by the ISNCSCI. However, a large subgroup of the sample presented with little or no upper limb sensation, and strength on the GRASSP detected areas of innervation.

To ensure a more accurate strength test, more than one muscle per myotome was incorporated. Although the sample is small when grouped by ISNCSCI level, the specific muscles, namely the anterior deltoid (C5), extensor digitorum, flexor pollicis longus (C8), and first dorsal interosseus (T1), provided important information regarding innervation. As expected, the greatest amount of discordance was level-specific $(\mathrm{C} 5, \mathrm{C} 8$, and T1), and predominantly was due to the abovementioned muscles. The added value of the additional elements to the sensation and strength testing showed that upper limb impairment was more accurately defined by the GRASSP than that attained using previous approaches. ISNCSCI sensory assessment failed to accurately represent the sensory status, as sensation from only the dorsal side of the hand was assessed. In addition, the use of light touch and pinprick as the test modalities in the ISNCSCI has the potential of adding response variation by the individual being assessed. The GRASSP sensation test accounts for these two factors by including palmar test locations, and using a sensory test (SWM) which is well calibrated, and which reduces the opportunity for the individual to vary their responses. Furthermore, the ISNCSCI motor assessment inflates motor impairment (i.e., individuals appear more impaired than they really are), due to underrepresentation of muscles at the myotomal level.

Concurrent validity of a measure is determined by comparing a new test to related, existing measures in the field. Since a pre-exisiting adequate upper limb measure of impairment for tetraplegia was not available, the best available functional measures were used. Concurrent validity is determined when a new test shows the anticipated associations to the comparator measures used. The subtests within the GRASSP show positive and significant associations with all the functional measures used in the field. The subtests that reflect impairment show moderate associations with the SCIM and CUE questionnaire, and the subtests that measure impairment within a functional paradigm (prehension) show stronger associations with the SCIM and CUE questionnaire. Interestingly, self perception of function had the highest association with the GRASSP, lending support to the theory that patients can detect meaningfulness during reporting based on their perceptions of their ability. As the secondary measures become more specific to the upper limb rather than the whole person, the association becomes stronger for all domains. Subtests within the GRASSP demonstrate moderate to substantial concurrent validity with the SCIM and the CUE questionnaire, indicating a positive relationship between impairment, function, and independence.

The GRASSP version 1.0 is at a stage where it can be added to protocols as part of the assessment battery to enhance the information gathered specific to the upper limb. The GRASSP provides five subtest scores for each upper limb. The five separate scores characterize the upper limb by presenting sensory and motor deficits, and the impact of these deficits on the performance of prehension/function. The GRASSP defines the core impairments of the upper limb and how they affect hand function. In other words the prehension testing allows the assessor to determine what role (impact) sensation and strength have on an individual's ability to perform functional tasks, clarifying the cause of the functional deficit, and in turn informing clinical decision making for targeted interventions, such as functional electrical stimulation to generate muscle force to replace motor function, or vision for determining juxtaposition of the fingertips to compensate for lack of the ability to sensate during pinch. The GRASSP is intended for use with the acute, post-acute, and chronic tetraplegic individual. Repeated use over time should provide a recovery profile of all three domains, whether due to spontaneous recovery, pharmacological interventions, or restorative upper limb therapies. Not all changes in neurological status are large enough to be realized functionally, so the GRASSP has been designed to capture changes in neurological recovery rather than function alone. Moreover, in some cases improved function may not be associated with neurological recovery, but rather with compensation. Furthermore, functional measures used in interventional studies have not always been sensitive enough to detect small gains. Therefore, measuring impairment over the post-injury course is imperative to determine how much neurological recovery is actually occurring, and how it relates to functionality. 
Table 9. GRASSP Subtest Scores of Four Representative Examples (Right Side Data Only)

\begin{tabular}{lcccrr}
\hline ISNCSCI & \multicolumn{4}{c}{ GRASSP subtest scores } \\
\cline { 2 - 6 } $\begin{array}{l}\text { Sensory/ } \\
\text { motor AIS }\end{array}$ & $\begin{array}{c}\text { Dorsal } \\
\text { sensation 0-12 }\end{array}$ & $\begin{array}{c}\text { Palmar } \\
\text { sensation 0-12 }\end{array}$ & $\begin{array}{c}\text { Strength } \\
0-50\end{array}$ & $\begin{array}{c}\text { Prehension } \\
\text { ability 0-12 }\end{array}$ & $\begin{array}{c}\text { Prehension } \\
\text { performance 0-30 }\end{array}$ \\
\hline C5/C4/A & 4 & 3 & 5 & 0 & 0 \\
C7/C6/A & 6 & 9 & 23 & 10 & 21 \\
C5/C6/D & 10 & 10 & 26 & 5 & 16 \\
C4/T1/D & 12 & 12 & 45 & 12 & 27
\end{tabular}

ISNCSCI, International Standards of Neurological Classification of Spinal Cord Injury; sensory, ISNCSCI sensory level; motor, ISNCSCI motor level; AIS, American Spinal Cord Injury Association impairment scale; GRASSP, Graded Redefined Assessment of Strength Sensibility and Prehension.

The limitations of the current study are largely attributed to sample selection and study design. We tested the GRASSP with a sample of individuals with chronic SCI in order to diminish the effects of maturation when establishing reliability, thereby ensuring that the individuals in the sample were considered neurologically stable per the criteria of Wright and Feinstein (1992). The strength of the relationship between impairment and function may not be as robust in more acute samples. Furthermore, to date we have not demonstrated responsiveness. Our current work consists of a longitudinal study in which we will examine the temporal changes in the three domains to determine their relationship to functional outcome in the upper limb, and to assess whether the GRASSP demonstrates responsiveness over the time frame of the established recovery phase reported to date.

We have shown the reliability of the GRASSP, demonstrating its use as a repeatable measure by multiple assessors. The strategies employed to reduce inter-observer variability, including the engagement of experienced clinicians as examiners (occupational therapists and physiotherapists) with SCI experience, and the comprehensiveness of the training using SCI patients as models, undoubtedly contributed to our positive findings. Having experienced clinicians involved in the assessment of neurological status obviously improves consistency.

The results have provided the necessary evidence to confirm that the GRASSP has the psychometric properties of reliability and validity, and is ready for widespread use in cross-sectional studies. The approach to scoring will continue to be five subtest scores, as there is a demonstrated importance in having a set of scores that report impairments in the different domains tested by the GRASSP, and presenting five subtest total scores on a radar graph will remain the approach to represent the GRASSP for clarity of interpretation at this stage of its development. Table 9 contains the GRASSP subscores for four representative examples from the sample; ISNCSCI sensory and motor levels, and classification for the right side only have been used to define the individuals as well.

\section{Acknowledgments}

This research was funded by the Christopher and Dana Reeve Foundation and the Rick Hansen Foundation. Sukhvinder Kalsi-Ryan was supported by a graduate student award from the Ontario Neurotrauma Foundation and the
Toronto Rehabilitation Institute Student Scholarship Fund. Dr. Beaton was supported by a CIHR New Investigator's award during the conduct of this study. The International GRASSP Research and Design Team provided assistance with organization of the study. We would like to acknowledge the GRASSP Cross Sectional Study Group for their commitment to the study and for providing assistance in implementing the study at their specific centers. The individuals in the GRASSP Cross Sectional Study Group were: Kimberley Eberhardt and Rebecca Ozelie at the Rehabilitation Institute of Chicago; Megan Watts and Rob Corcoran at Vancouver Coastal Health; Marlene Adams, Sylvia Coates, and Abigail Dry at the Toronto Rehabilitation Institute; Gina Cooke at the Magee Rehabilitation-Regional Spinal Cord Injury Center of the Delaware Valley; Christina Robert at Balgrist, Zurich; Martha Horn and Simone Hirsch at the Trauma Center, Murnau; and Kristin Lorenz and Petra Schatz at Hohe Warte, Bayreuth.

\section{Author Disclosure Statement}

No conflicting financial interests exist.

\section{References}

Anderson, K.D. (2004). Targeting recovery: Priorities of the spinal cord-injured population. J. Neurotrauma 21, 1371-1383.

Brand, P.W., and Hollister, A.M. (1991). Clinical Mechanics of the Hand, 3rd ed. Mosby: St. Louis.

Brandsma, J.W., Schreurders, T.A., Birke, J.A., and Oostendorp, R. (1995). Manual muscle testing: intraobserver and interobserver reliabilities for the intrinsic muscles of the hand. J. Hand Ther. 8, 185-190.

Catz, A., and Itzkovich, M. (2007). Spinal Cord Independence Measure: Comprehensive ability rating scale for the spinal cord lesion patient. J. Rehab. Res. Dev. 44, 65-68.

Catz, A., Greenberg, E., Itzkovich, M., Bluvshtein, V., Ronen, J., and Gelernter, I. (2004). A new instrument for outcome assessment in rehabilitation medicine: Spinal cord injury ability realization measurement index. Arch. Phys. Med. Rehab. 85, 399-404.

Daniels, L., and Worthingham, C. (1995). Daniels and Worthingham's Muscle Testing: Techniques of Manual Examination, 6th ed. WB Saunders Co.: Washington.

DeVivo, M.J. (1997). Causes and costs of spinal cord injury in the United States. Spinal Cord 35, 809-813.

Doner, A., and Eliasziw, M. (1987). Sample size requirements for reliability studies. Statistics Med. 5, 441-448. 
Dunn, J.A., Sinnott, K.A., Bryden, A.M., Connolly, S.J., and Rothwell, A.G. (2008). Measurement issues related to upper limb interventions in persons who have tetraplegia. Hand Clinics 24, 161-168.

Ellaway, P.H., Kuppuswamy, A., Balasubramaniam, A., Maksimovic, R., Gall, A., Craggs, M.D., Kowalczewski, J., Conway, B.A., Galen, S., Catton, C.J., Allan, D.B., Curt, A., Wirth, B., and van Hedel, H. (2010). Development of quantitative and sensitive assessments of physiological and functional outcome during recovery from spinal cord injury: Clinical initiative. Brain Res. Bull. [Epub before print].

Fujiwara, T., Hara, Y., Akaboshi, K., and Chino, N. (1999). Relationship between shoulder muscle strength and functional independence measure (FIM), score among C6 tetraplegics. Spinal Cord 37, 58-61.

Kalsi-Ryan, S., Curt, A., Fehlings, M.G., and Verrier, M.C. (2009). Assessment of the hand in tetraplegia using the Graded Redefined Assessment of Strength Sensibility and Prehension (GRASSP): Impairment versus function. Top. SCI Rehab. 14, 34-46.

Kohlmeyer, K.M., Hill, J.P., Yarkony, G.M., and Jaegar, R.J. (1996). Electrical stimulation and biofeedback effect on recovery of tenodesis grasp: A controlled study. Arch. Phys. Med. Rehab. 77, 702-706.

Landis, J.R., and Koch, G.G. (1977). The measurement of observer agreement for categorical data. Biometrics 33, 159-174.

Mackin, E., Callahan, A., Skiver, T., Schneider, L., and Osterman, A. (2003). Hunter-Mackin Callahan Rehabilitation of the Hand and Upper Extremity, 5th ed. Mosby: St. Louis.

Marino, R.J. (2000). American Spinal Injury Association: International Standards of Neurological Classification of the Spinal Cord Injury. Atlanta, Georgia. Reprinted 2008.

Marino, R.J., Ditunno, J.F., Donovan, W.H., and Maynard, F. (1999). Neurological recovery after traumatic spinal cord injury: data from the model spinal cord injury systems. Arch. Phys. Med. Rehab. 80, 1391-1396.

Marino, R.J., Shea, J.A., and Stineman, M.G. (1998). The Capabilities of the Upper Extremity instrument reliability and validity of a measure of functional limitation in tetraplegia. Arch. Phys. Med. Rehab. 79, 1512-1521.

Miller, W.C., Aubut, J.L., Curt, A., Connolly, S.J., Elliott, S., Hsieh, J.T.C., Mortenson, W.B., Noonan, V.K., Noreau, L., Orenczuk, S.G., Sakakibara, B.M., Sawatzky, B., Steeves, J., Wilkinson, S., and Wolfe, D. (2008). SCIRE: Spinal Cord Injury Rehabilitation Evidence Version 2.0. Chapter 25, Outcome Measures.

Moore, K.L. (1985). Clinically Oriented Anatomy, 2nd ed. Williams and Wilkins: Baltimore.

Nunnally, J.C., and Bernstein, I.H. (1996). In Psychometric Theory. McGraw-Hill: Toronto.

D.L. Patrick, D.L. and P. Erickson, (eds). (1993). Health Status and Health Policy Allocation Resource to Health Care. Oxford University Press: New York.

Portney, L.G., and Watkin, M.P. (2000). Statistical measures of reliability, in: Foundations of Clinical Research: Applications to Practice, 2nd ed. Appleton and Lange: Norwich.

Popovic, M.R., Thrasher, T.A., Adams, M.A., Takes, V., Zivanovic, V., and Tonack, M.I. (2006). Functional electrical therapy: retraining grasping in spinal cord injury. Spinal Cord 44, 143-151.
Priebe, M., and Waring, W.P. (1991). The interobserver reliability of the revised American Spinal Injury Association standards for neurological classification of spinal injury patients. Am. J. Phys. Med. Rehabil. 70, 268-270.

Prochazka, A., Gauthier, M., Wieler, M., and Kenwell, Z. (1997). The bionic glove: an electrical stimulator garment that provides controlled grasp and hand opening in quadriplegia. Arch. Phys. Med. Rehab. 78, 608-614.

Rudhe, C., and van Hedel, H. (2009). Upper extremity function in persons with tetraplegia: Relationships between strength, capacity and the Spinal Cord Independence Measure. Neurorehab. Neural Repair 23, 413-421.

Sekhon, L., and Fehlings, M.G. (2001). Epidemiology, demographics, and pathophysiology of acute spinal cord injury. Spine 26, S2-S12.

Shumway-Cook, A., and Woollacott, M.H. (2007). Motor Control: Translating Research Into Clinical Practice, 3rd ed. Lippincott, Williams \& Wilkins: St. Louis.

Snoek, G.J., Ijzerman, M.J., Post, M.W., Stiggelbout, A.M., Roach, M.J., and Zivold, G. (2005). Choice-based evaluation for the improvement of upper-extremity function compared with other impairments in tetraplegia. Arch. Phys. Med. Rehab. 86, 1623-1630.

Sollerman, C., and Ejeskar, A. (1995). Sollerman Hand Function Test: A standardized method and its use in tetraplegic patients. Scand. J. Plast. Reconstruct. Hand Surg. 29, 167-176.

Steeves, J.D., Lammertse, D., Curt, A., Fawcett, J.W., Tuszynski, M.H., Ditunno, J.F., Ellaway, P.H., Fehlings, M.G., Guest, J.D., Kleitman, N., Bartlett, P.F., Blight, A.R., Dietz, V., Dobkin, B.H., Grossman, R., Short, D., Nakamura, M., Coleman, W.P., Gaviria, M., and Privat, A. (2007). Guidelines for the conduct of clinical trials for spinal cord injury (SCI) as developed by the ICCP panel: clinical trial outcome measures. Spinal Cord 45, 206-221.

Streiner, D.L., and Norman, G.L. (1995a). Reliability, in: Health Measurement Scales: A Practical Guide to Their Development and Use, 2nd ed. Oxford University Press: New York, pps. 104-127. Streiner, D.L., and Norman, G.L. (1995b). Validity, in: Health Measurement Scales: A Practical Guide to Their Development and Use, 2nd ed. Oxford University Press: New York, pps. 144-162.

Taylor, P., Esnouf, J., and Hobby, J. (2002). The functional impact of the Freehand System on Tetraplegic hand function. Clinical results. Spinal Cord 40, 560-566.

van Hedel, H. and the EMSCI Study Group. (2009). Gait speed in relation to categories of functional ambulation after spinal cord injury. Neurorehabil. Neural Repair 23, 117-124.

van Tuijl, J.H., Janssen-Potten, Y.J.M., and Seelen, H.A.M. (2002). Evaluation of upper extremity motor function tests in tetraplegics. Spinal Cord 40, 51-64.

Wright, J.G., and Feinstein, A.R. (1992). Improving the reliability of orthopaedic measurements. J. Bone Joint Surg. 74, 287-291.

Address correspondence to: Sukhvinder Kalsi-Ryan, BScPT, M.Sc., Ph.D. Toronto Western Hospital McL 12th Floor, Room 407 399 Bathurst Street Toronto, ON, M5G 2S8

Email: Sukhvinder.Kalsi-Ryan@uhn.on.ca 